\title{
THE CRITICAL EXPONENTS OF THE DECONFINEMENT TRANSITION IN SU(2) LATTICE GAUGE THEORY
}

\author{
J. ENGELS ${ }^{\mathrm{a}, \mathrm{b}}$, J. JERSÁK $^{\mathrm{c}}, \mathrm{K}^{\mathrm{K}}$ KANAYA ${ }^{\mathrm{c}}$, E. LAERMANN ${ }^{\mathrm{d}}$, C.B. LANG ${ }^{\mathrm{e}}$, \\ T. NEUHAUS ${ }^{a}$ and H. SATZ ${ }^{b, f}$
}

Received 1 September 1986

\begin{abstract}
The behaviour of the deconfinement order parameter, the susceptibility, the correlation length and the energy density of the SU(2) Yang-Mills system is studied in the neighbourhood of the deconfinement transition on large lattices $\left(18^{3} \times 3,4,5\right.$ and $\left.16^{3} \times 4,5,6\right)$. We check the critical exponents both below and above the transition by fits including corrections-to-scaling terms and find very good agreement with the universality predictions from the $Z_{2}$ spin system. With the predicted exponent $\beta$ as input, precise values for the critical temperature are found; they are confirmed by the energy density results.
\end{abstract}

\section{Introduction}

The deconfinement transition of $\mathrm{SU}(N)$ lattice gauge theory at finite temperature is related to the global center $Z_{N}$ symmetry of the theory [1,2]. In the deconfinement phase the global symmetry is spontaneously broken, in the confinement regime the symmetry is restored. The expectation value of the Polyakov loop (thermal Wilson line) is the corresponding order parameter for the deconfinement transition [3-5]. It measures the free energy $F_{\mathrm{q}}$ of a single static (infinite mass) quark at temperature $T$

$$
\langle L\rangle \sim \exp \left(-\frac{F_{\mathrm{q}}}{T}\right)
$$

and is zero when $F_{\mathrm{q}}$ is infinite, i.e. in the confinement region, but finite in the deconfined phase. For a second-order phase transition, like the one in SU(2), the situation is then analogous to that of the Ising model, where below some critical

${ }^{a}$ Supercomputer Computations Research Institute, Florida State University, Tallahassee, Florida 32306, USA.

${ }^{b}$ Fakultät für Physik, Universität Bielefeld, 4800 Bielefeld, Fed. Rep. Germany.

${ }^{c}$ Institut für Theoretische Physik E, RWTH Aachen, 5100 Aachen, Fed. Rep. Germany.

d Institut für Theoretische Physik, Bergische Universität, 5600 Wuppertal, Fed. Rep. Germany.

' Institut für Theoretische Physik, Universität Graz, 8010 Graz, Austria.

${ }^{f}$ Physics Department, Brookhaven National Laboratory, Upton, New York 11973, USA. 
Definitions of the critical exponents $\alpha, \beta, \gamma, \nu$

\begin{tabular}{|c|c|c|}
\hline $\begin{array}{l}\text { spontaneous magnetization } \\
\text { susceptibility }\end{array}$ & $\begin{aligned}\langle L\rangle & \sim\left(T-T_{\mathrm{c}}\right)^{\beta} \\
\chi & =N_{\sigma}^{3}\left(\left\langle L^{2}\right\rangle-\langle L\rangle^{2}\right) \\
& \sim\left|T-T_{\mathrm{c}}\right|-\gamma\end{aligned}$ & $\begin{array}{l}\text { as } T \rightarrow T_{\mathrm{c}}+ \\
\text { as } T \rightarrow T_{\mathrm{c}}\end{array}$ \\
\hline specific heat & $\begin{aligned} C_{\mathrm{v}} & =T^{2} \frac{\partial^{2} \ln Z}{\partial T^{2}} \\
& \sim\left|T-T_{\mathrm{c}}\right|^{-\alpha}\end{aligned}$ & as $T \rightarrow T_{\mathrm{c}}$ \\
\hline correlation length & $\begin{array}{l}\xi \sim\left|T-T_{\mathrm{c}}\right|^{-\nu} \\
\xi=T / \sigma\end{array}$ & $\begin{array}{l}\text { as } T \rightarrow T_{\mathrm{c}} \\
\text { if } T<T_{\mathrm{c}}\end{array}$ \\
\hline
\end{tabular}

temperature a spontaneous magnetization exists, which in the neighbourhood of the critical point is described by

$$
M \sim\left(T_{\mathrm{c}}-T\right)^{\beta}
$$

where $\beta$ is the critical exponent of the order parameter. There is one difference in $\mathrm{SU}(2)$ lattice gauge theory compared to ferromagnetism: the order parameter is zero in the low-temperature phase and non-zero at high temperatures. Apart from that, all the corresponding physical quantities and their respective critical exponents exist. They are shown in table 1.

In the strong coupling limit it is possible to perform all integrations in the path integral formula for the partition function, except those for the Wilson line operators [6]; one is then left with an effective theory of Wilson lines, which has the same structure as a $Z_{N}$ spin theory of the same spatial dimension [5-8]. This was generalized by Svetitsky and Yaffe [7] to arbitrary couplings as a universality conjecture, implying the same critical behaviour for $\mathrm{SU}(N)$ gauge theory at the deconfinement transition and $Z_{N}$ spin theory at the order-disorder transition, if both transitions are continuous. As a consequence, the SU(2) lattice gauge theory in $3+1$ dimensions, which we are about to consider in this paper, should have the same critical exponents as the three-dimensional Ising model. The critical exponents of the latter theory are known to a high degree of precision from approximate theoretical methods [9-11] as well as Monte Carlo simulations [12].

In this paper we shall check these predictions (see table 2) both below and above the critical temperature by appropriate fits. The critical coupling or temperature is one of the parameters obtained through these fits. This will allow a test of the asymptotic scaling relation as obtained from the renormalization group equation (RGE)

$$
a\left(g^{2}\right) \Lambda_{\mathrm{L}}=\exp \left\{-\frac{12 \pi^{2}}{11 g^{2}}+\frac{51}{121} \ln \left(\frac{24 \pi^{2}}{11 g^{2}}\right)\right\}
$$


TABLE 2

Critical exponents for the 3-dimensional Ising model

\begin{tabular}{lcccc}
\hline Method & $\beta$ & $\gamma$ & $\nu$ & $\alpha$ \\
\hline$\varepsilon$-expansion [10] & $0.3265 \pm 0.0025$ & $1.239 \pm 0.004$ & $0.6305 \pm 0.0025$ & \\
$\begin{array}{c}\text { renormalization } \\
\text { group estimates [9] }\end{array}$ & $0.3250 \pm 0.0015$ & $1.241 \pm 0.002$ & $0.6300 \pm 0.0015$ & \\
$\begin{array}{c}\text { high temperature } \\
\text { series [11] }\end{array}$ & $1.2395 \pm 0.0004$ & $0.632 \pm 0.001$ & $0.105 \pm 0.007$ \\
$\begin{array}{c}\text { Monte Carlo } \\
\text { simulation [12] }\end{array}$ & $1.240 \pm 0.002$ & $0.638 \pm 0.009$ & $0.09 \pm 0.01$ \\
\hline
\end{tabular}

for different $N_{\tau}$ (number of lattice points in time direction) with

$$
T_{\mathrm{c}}=\frac{1}{N_{\tau} a\left(g_{\mathrm{c}}^{2}\right)} .
$$

There have been several attempts $[5,13-15]$ to determine the critical exponent $\beta$ of the SU(2) order parameter. This was usually done with a one term fit on either relatively small lattices and/or not too high statistics. Whereas the earliest study on a $7^{3} \times 3$ lattice quotes, $\beta=0.207 \pm 0.008$ [5], the other authors report agreement $[13,14]$ or at least compatibility [15] with $\beta=0.33$. Apart from one paper [16] (in which some results for the correlation length are given), no effort was made, as far as we know, to determine the other critical exponents or to compare them with the exponents of the Ising model. We shall here present high precision measurements on large lattices, including not only results for the order parameter, but also the susceptibility, correlation length and and energy density.

The paper is organized as follows. In the second section we shall present the data for the order parameter and their analysis. The next section will contain the results for the energy density. Sect. 4 contains a discussion of the susceptibility and the correlation length measurements both above and below the transition point. We summarize the findings in sect. 5 .

\section{The order parameter}

The Polyakov loop for $\mathrm{SU}(2)$ gauge theory on an $N_{\mathrm{o}}^{3} \times N_{\tau}$ lattice is defined as

$$
L(\boldsymbol{x})=\frac{1}{2} \operatorname{Tr} \prod_{\tau=1}^{N_{\tau}} U_{\tau, x ; 0},
$$

where $U_{x ; 0}$ are the $\mathrm{SU}(2)$ link matrices at four-position $x$ in the time direction. We 
use the standard Wilson action

$$
S(U)=\frac{4}{g^{2}} \sum_{\mathrm{P}} \operatorname{Tr} U_{\mathrm{p}} ;
$$

here $U_{\mathrm{P}}$ is the product of link operators around a plaquette.

The computation of the average value of the Polyakov loop on a finite lattice is straightforward; however, there are difficulties arising either from the finite size $N_{\sigma}$ and/or the existence of the deconfinement transition itself. On a finite lattice flips among different equivalent states occur, in our case $L \rightarrow-L$, which for an infinite number of lattice sweeps will lead to a vanishing average value even in the deconfinement region. We therefore use the modulus of the lattice average

$$
\bar{L}=\left|\frac{1}{N_{\sigma}^{3}} \sum_{x} L(x)\right|,
$$

and define $\langle\bar{L}\rangle$ as the configuration average of $\bar{L}$. On the other hand, due to finite statistics and finite lattice size, the average value of the Polyakov loop will be non-zero also in the confined phase. Therefore, the exact location of the critical coupling has to be determined by some type of extrapolation. To assess the importance of such finite size effects we have calculated $\langle\bar{L}\rangle$ on an $8^{3} \times 3$ and an $18^{3} \times 3$ lattice in the neighbourhood of the deconfinement transition. The results in fig. 1 show clearly the necessity to work on lattices as large as possible, at least when

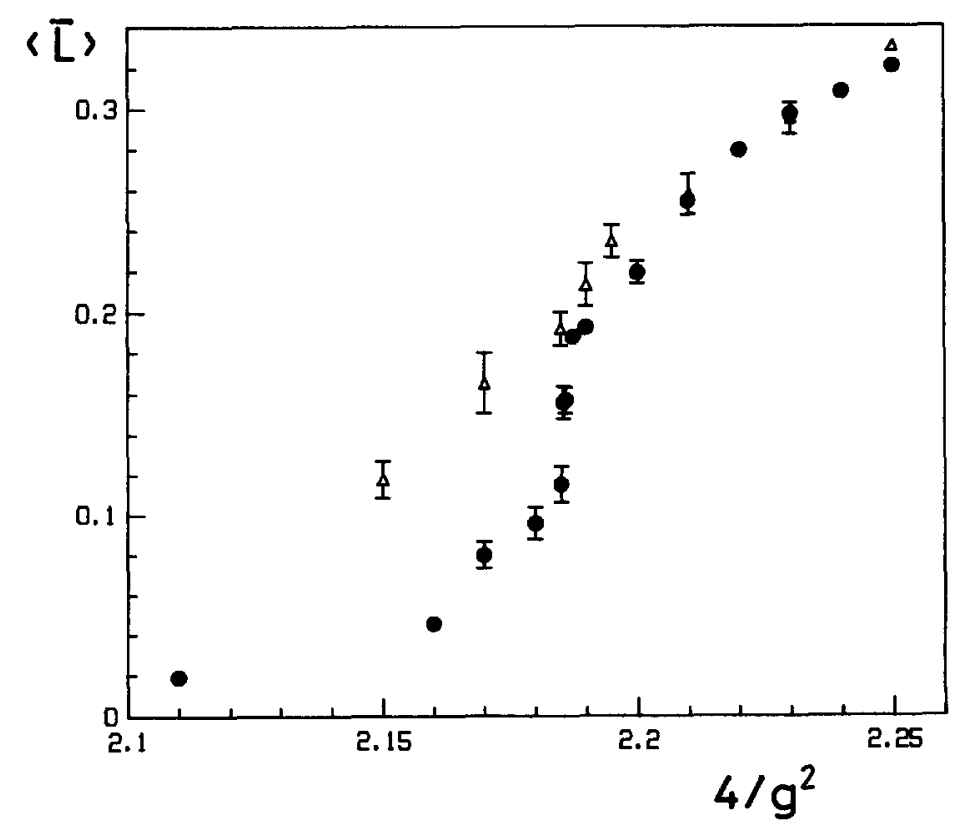

Fig. 1. Order parameter $\langle\bar{L}\rangle$ versus $4 / g^{2}$ on an $8^{3} \times 3(\Delta)$ and an $18^{3} \times 3(\bullet)$ lattice. 


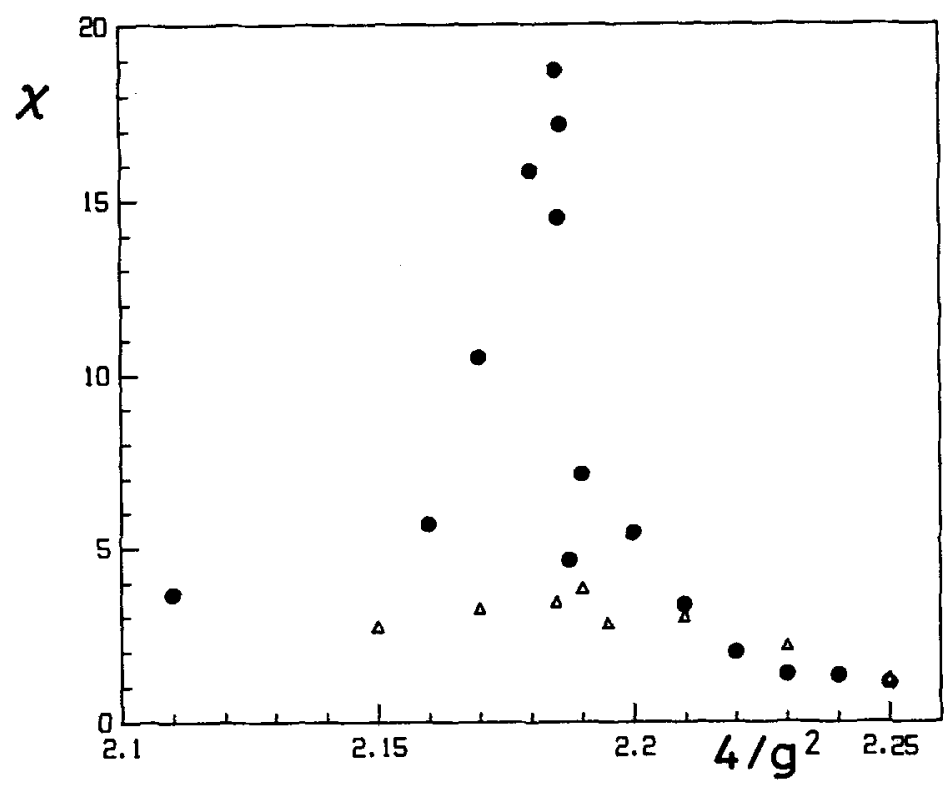

Fig. 2. Susceptibility versus $4 / g^{2}$ on the same lattices as in fig. 1 .

one wants to examine the transition region. The differences between the two lattices are even more dramatic for the susceptibility, as can be seen in fig. 2 .

All our data were obtained from a one hit Metropolis program using the icosahedral subgroup of $\mathrm{SU}(2)$, starting measurements after 500 thermalization sweeps from cold configurations. Apart from the correlation functions, which were computed only every fifth update, all other quantities have been calculated every sweep. The error analysis of the raw data is described in the appendix. We have three sets of data: on an $8^{3} \times 3$ lattice with 20000 updates per point, on $18^{3} \times 3,4,5$ lattices with 20000-60000 updates per point and below the critical temperature on $16^{3} \times 4,5,6$ lattices with $29000-58500$ updates per point. These large numbers of sweeps had to be performed because of the "critical slowing down" effect of the Metropolis method on large lattices and because of the increase of the correlation length, when a second-order transition point is approached. Both effects correlate successive updates and more sweeps are necessary to produce independent link configurations.

In fig. 3, the average Polyakov loop $\langle\bar{L}\rangle$ values on an $18^{3} \times 3$ lattice are shown together with a fit of the form

$$
\langle\bar{L}\rangle=A\left(4 / g^{2}-4 / g_{c}^{2}\right)^{\beta}\left(1+B\left(4 / g^{2}-4 / g_{c}^{2}\right)^{\theta}\right)
$$

We have chosen $4 / g^{2}$ as variable in (8) instead of $T$, since a fit in $T$ would assume 


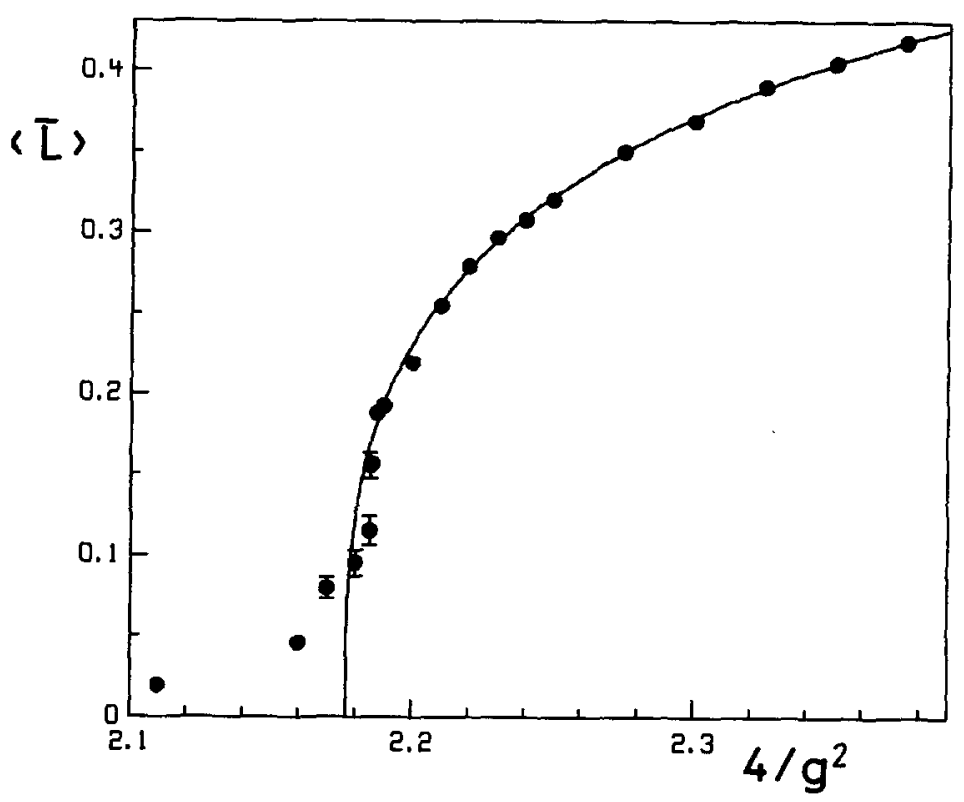

Fig. 3. Order parameter $\langle\bar{L}\rangle$ versus $4 / g^{2}$ on the $18^{3} \times 3$ lattice. The curve is the best fit of $\langle\bar{L}\rangle$ according to eq. (8), taking all points with $4 / g^{2} \geqslant 2.1855$ into account.

the asymptotic scaling relation, eq. (3), which we want to test. It will be used later for fits below $T_{\mathrm{c}}$. In the neighbourhood of the transition the change of variables will only affect the parameters $A$ and $B$, not the critical exponents. A correction to the leading term has to be included in the fit, because the range of validity of the leading term approximation is not known. With the form (8) excellent fits are obtained, which only differ very close to the critical temperature. Moreover, the exponent $\theta$ is also universal and known from the Ising model to be about 0.5

TABLE 3

Best fits to $\langle\bar{L}\rangle=A\left(4 / \mathrm{g}^{2}-4 / \mathrm{gc}_{\mathrm{c}}^{2}\right)^{\beta}\left(1+B\left(4 / \mathrm{g}^{2}-4 / \mathrm{g}_{\mathrm{c}}^{2}\right)^{0.5}\right)$

\begin{tabular}{cccccccc}
\hline Lattice & $A$ & $B$ & $4 / g_{\mathrm{c}}^{2}$ & $T_{\mathrm{c}} / \Lambda_{\mathrm{L}}$ & $\beta$ & $\chi^{2} / n_{\mathrm{f}}$ & $4 / g^{2}$ \\
\hline \multirow{2}{*}{$18^{3} \times 3$} & 0.860 & -0.369 & 2.1768 & 41.40 & 0.335 & 2.49 & $\geqslant 2.1855$ \\
& 0.856 & -0.364 & 2.1768 & 41.40 & $1 / 3$ & & \\
& 0.756 & -0.229 & 2.1795 & & 0.297 & 4.52 & $\geqslant 2.185$ \\
\multirow{2}{*}{$18^{3} \times 4$} & 0.860 & -0.372 & 2.1775 & & $1 / 3$ & & \\
& 0.728 & -0.451 & 2.2954 & 41.79 & 0.364 & 1.16 & $\geqslant 2.30$ \\
$18^{3} \times 5$ & 0.648 & -0.320 & 2.2964 & 41.89 & $1 / 3$ & & \\
& 0.358 & 0.170 & 2.3769 & 41.02 & 0.252 & 0.78 & $\geqslant 2.385$ \\
& 0.492 & -0.258 & 2.3726 & 40.58 & $1 / 3$ & & \\
& 0.624 & -0.493 & 2.3662 & & 0.409 & 1.07 & $\geqslant 2.375$ \\
& 0.468 & -0.156 & 2.3689 & & $1 / 3$ & & \\
\hline
\end{tabular}




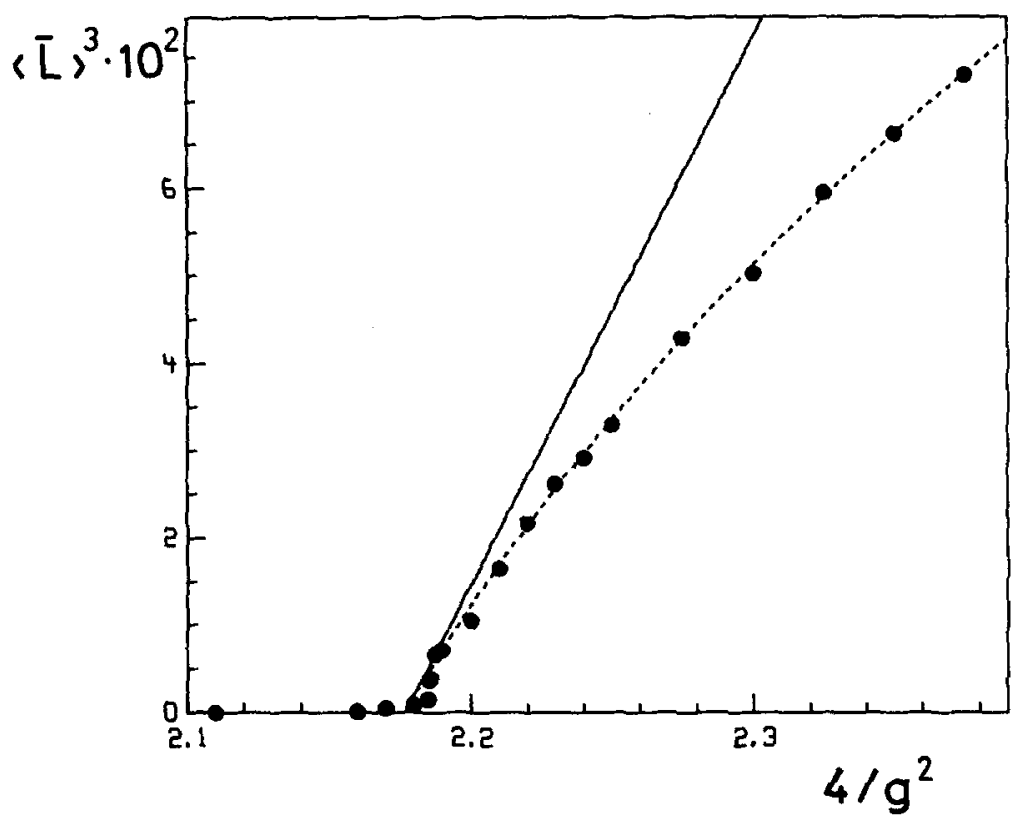

Fig. 4. $\langle\bar{L}\rangle^{3} \times 10^{2}$ versus $4 / g^{2}$ on the $18^{3} \times 3$ lattice. The dashed line is a fit (eq. (8)) with fixed $\beta=\frac{1}{3}$, the solid line its leading term.

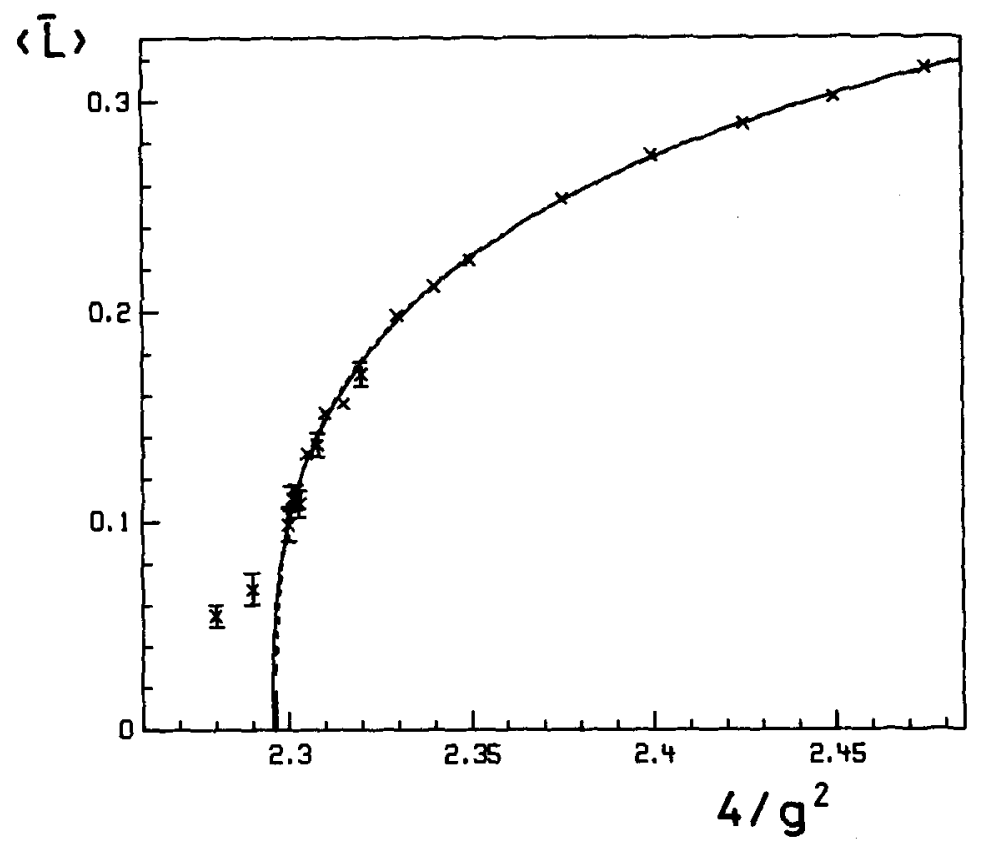

Fig. 5. As fig. 3, for the $18^{3} \times 4$ lattice. The solid line is the best fit for points with $4 / g^{2} \geq 2.30$, the dashed line the corresponding fit with fixed $\beta=\frac{1}{3}$. 
$[11,17]$. Due to the functional form of the fit, the parameters $4 / g_{c}^{2}$ and $\beta$ are strongly correlated - a slight change in $4 / g_{c}^{2}$ leads to a relatively large change in the critical exponent, whereas $\beta$ and $\theta$ are only weakly dependent on each other. To reduce the number of parameters we have therefore fixed $\theta$ in all fits to 0.5 .

Returning to fig. 3 , it is obvious that the points for $4 / g^{2} \leqslant 2.18$ are influenced by finite size effects and have to be excluded from the fits; however, for $4 / g^{2}=2.185$ this is not so clear. In table 3 the parameters of the best fits with and without this ambiguous point are given. We also performed fits with $\beta$ fixed to $\frac{1}{3}$ and show them with the data for $\langle\bar{L}\rangle^{3}$ in fig. 4. Evidently a fit with only the leading term would not be possible for all of our data and would lead to a too low value for $4 / g_{c}^{2}$.

The variation of the critical exponent within the $95 \%$ confidence level is smaller than the systematic error induced by the inclusion or not of the point $4 / g^{2}=2.185$ in the region, which is biased by finite size effects. We therefore conclude that the error in $\beta$ from the $18^{3} \times 3$ data is of the order of $10 \%$. In figs. 5 and 6 we show the corresponding plots and the best fits for $N_{\tau}=4$ and 5 . Whereas for $N_{\tau}=4$ we have no difficulty to exclude irrelevant points, it is essentially not possible to do this unambiguously for $N_{\tau}=5$, if the critical point is not known from other sources. Including or not the point $4 / \mathrm{g}^{2}=2.375$ for $N_{\tau}=5$ changes the best fit value for $\beta$ from 0.25 to 0.41 , i.e. we have a large systematic error. On the other hand the predicted value for $\beta$ is just the average of the two last numbers.

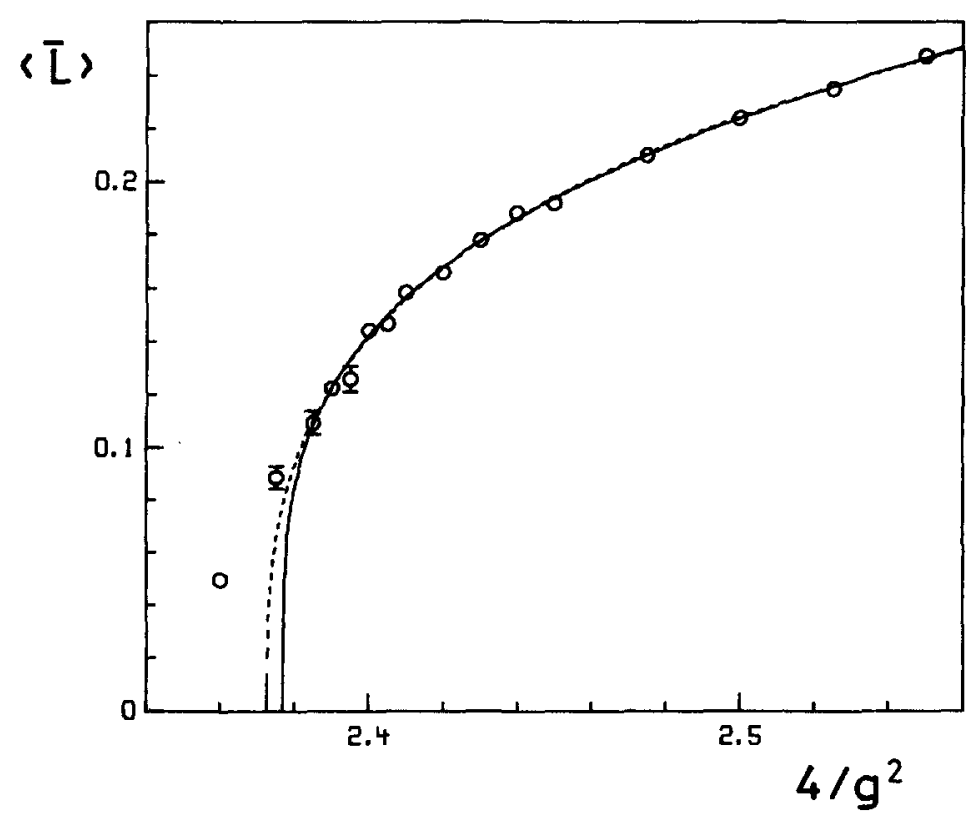

Fig. 6. As fig. 5 , for the $18^{3} \times 5$ lattice. For the fits all points with $4 / g^{2} \geqslant 2.385$ were used. 
We conclude that the expected value for the critical exponent $\beta$ is very well compatible with the data for $N_{\tau}=3,4$ and 5. One can now in turn use the prediction for $\beta$ to determine the critical coupling more accurately. The results are again given in table 3 and show that the $T_{\mathrm{c}}$ values calculated from eqs. (3) and (4) agree essentially for $N_{\tau}=3$ and 4 , whereas $T_{\mathrm{c}}\left(N_{\tau}=5\right)$ is slightly lower. This result is confirmed by the data for the energy density.

\section{The energy density}

In zeroth approximation the energy density for the SU(2) Yang-Mills system is given by

$$
\varepsilon_{0} a^{4}=\frac{12}{g^{2}}\left(P_{\sigma}-P_{\tau}\right)
$$

where $P_{\sigma}, P_{\tau}$ are the expectation values of the space-space and space-time plaquettes, respectively

$$
P_{\sigma, \tau}=1-\frac{1}{2}\left\langle\operatorname{Tr} U_{P_{\alpha, \tau}}\right\rangle .
$$

In the next order in $g^{2}$ the energy density is (for details see ref. [18])

$$
\begin{gathered}
\varepsilon a^{4}=\varepsilon_{0} a^{4}+\overline{c_{\sigma}}\left(P_{\mathrm{sym}}-P_{\sigma}\right)+\overline{c_{\tau}}\left(P_{\mathrm{sym}}-P_{\tau}\right) ; \\
\overline{c_{\sigma}}=1.368389, \quad \overline{c_{\tau}}=-0.810630 .
\end{gathered}
$$

Here $P_{\text {sym }}$ is the plaquette expectation value on a large symmetric $\left(N_{\sigma}=N_{\tau}\right)$ lattice. To calculate the correction terms we computed $P_{\text {sym }}$ on a $12^{4}$ lattice, where we found full agreement with the values of ref. [19]. We have plotted the data for $\varepsilon / \varepsilon_{\mathrm{SB}}$ in fig. 7. The quantity $\varepsilon_{S B}$ is the energy density for an ideal gas of gluons on the same size lattice [20] - the Stefan-Boltzmann energy density. We see in fig. 7 a rapid change in $\varepsilon / \varepsilon_{\mathrm{SB}}$ due to the deconfinement transition. Shortly after the transition the energy density is already close to the Stefan-Boltzmann value. Note, that since

$$
a^{4}=\frac{1}{N_{\tau}^{4} T^{4}},
$$

the Monte Carlo result for $\varepsilon a^{4}$ decreases with $N_{\tau}^{-4}$, so that an evaluation of the energy density on large $N_{\tau}$ lattices requires very high statistics. As $\varepsilon / \varepsilon_{\mathrm{SB}}$ is a dimensionless physical quantity, the same results should be obtained independent of $N_{\tau}$, if we plot them as a function of $T$. This is done in fig. 8 , assuming the asymptotic scaling relation (3). One observes, that the data for $N_{\tau}=3$ and 4 are scaling, whereas the data for $N_{\tau}=5$ are shifted with respect to the two other data sets by about $1.5 \Lambda_{\mathrm{L}}$ in temperature. This shift corresponds to the one observed already in the determination of the critical temperature from the order parameter 


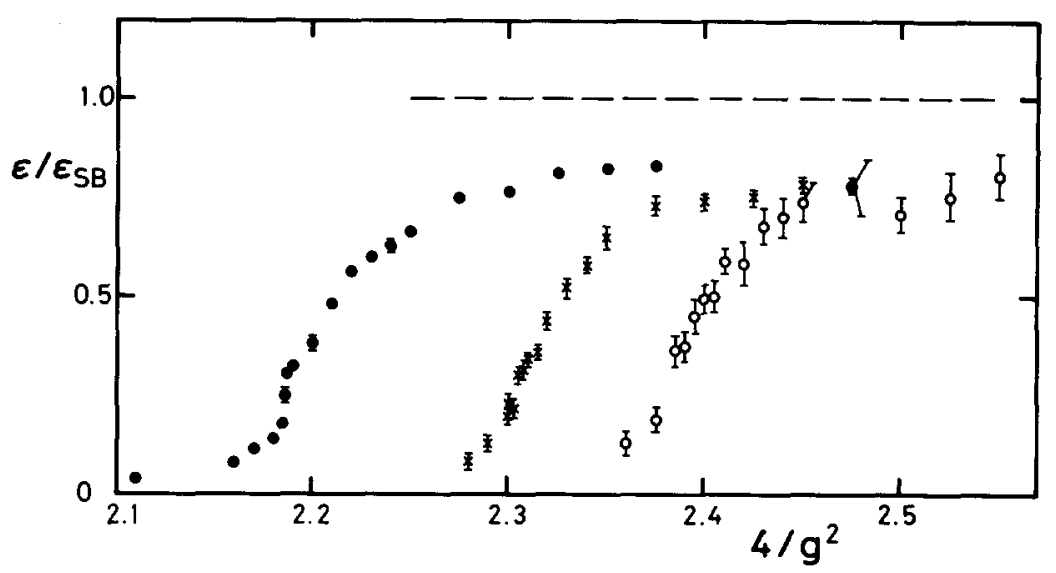

Fig. 7. Energy density divided by its Stefan-Boltzmann value on the $18^{3} \times 3(\bullet), 18^{3} \times 4(\times)$ and $18^{3} \times 5(0)$ lattices versus $4 / \mathrm{g}^{2}$.

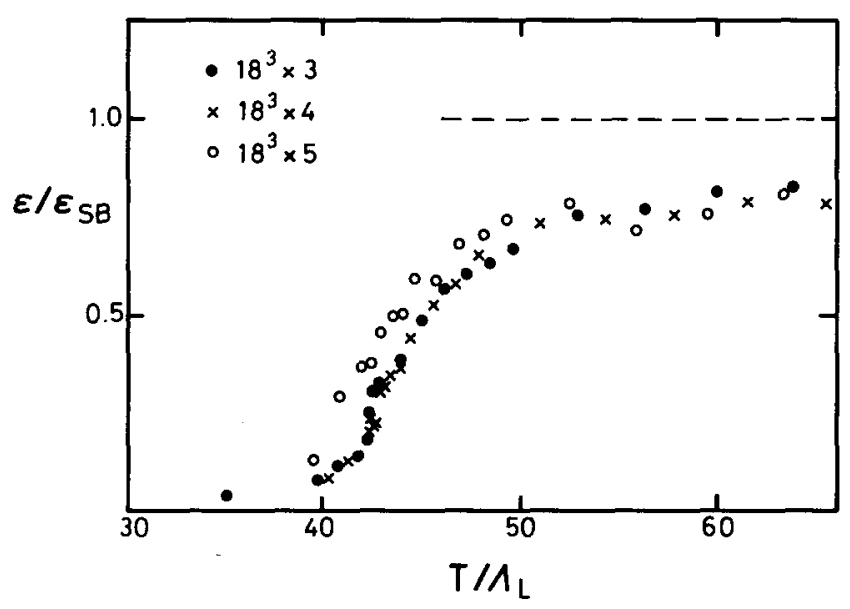

Fig. 8. As fig. 7, but versus temperature. For clarity the error bars have been omitted.

fits. However, apart from this shift in $T$, which indicates a deviation from the asymptotic scaling relation (3), we find the same functional behaviour of the energy density.

\section{Correlation length and susceptibility}

The connected correlation function is defined by

$$
\Gamma(x)=\langle L(0) L(x)\rangle-\langle\bar{L}\rangle^{2},
$$


where the subtraction of $\langle\bar{L}\rangle^{2}$ assures that $\Gamma(x)$ vanishes for $r=|x| \rightarrow \infty$ also in the deconfinement region. Since

$$
\lim _{r \rightarrow \infty}\langle L(0) L(r)\rangle=\langle\bar{L}\rangle^{2},
$$

it is indeed the configuration average of the absolute value $\bar{L}$ of the lattice average which has to be subtracted [21]. For large $r, \Gamma(r)$ decreases exponentially on either side of the transition point

$$
\Gamma(r) \sim \mathrm{e}^{-r / \xi},
$$

with $\xi$ denoting the correlation length; it diverges at $T_{\mathrm{c}}$. We have measured $\langle L(0) L(x)\rangle$ only parallel to the coordinate axes, not in the diagonals; moreover, since we use periodic boundary conditions also in the space-like directions, $\langle L(0) L(x)\rangle$ contains on a finite lattice contributions both from the separation $r$ and from $N_{\sigma} a-r$.

The relation between $\Gamma(x)$ and the interaction between the two static quarks given by the Polyakov loops $L(0)$ and $L(x)$ in $\langle L(0) L(x)\rangle$ is studied in ref. [21]. From this we expect the form

$$
\Gamma(r)=\frac{1}{r} \exp \left\{-c_{0}-c_{1} / r-r / \xi\right\}
$$

for the correlation function. For $T<T_{\mathrm{c}}$

$$
\xi^{-1}=\sigma / T
$$

measures the string tension $\sigma$, which vanishes at $T_{\mathrm{c}}$. The term $c_{1} / r$ contains the Coulomb interaction as well as the effect of string fluctuations [22], while $c_{0}$ fixes the overall normalization. In the high temperature region, $T>T_{c}, \xi$ is related to the gluonic colour screening radius $r_{\mathrm{D}}$

$$
\xi^{-1} \simeq r_{\mathrm{D}}
$$

that $1 / r$ factor in front of the exponential is now due to the Coulomb potential (one-gluon exchange) and $c_{1}$ is zero. We have thus fitted our results for $\Gamma(r)$ to the form

$$
\Gamma(r)=\frac{\mathrm{e}^{-N_{r} b(r)}}{r}+\frac{\mathrm{e}^{-N_{r} b\left(N_{o} a-r\right)}}{N_{\sigma} a-r},
$$

with

$$
b(r)=\overline{c_{0}}+\frac{\overline{c_{1}}}{r}+\overline{c_{2}} r .
$$

The second term in eq. (20) takes into account the mentioned spatial periodicity. 
The susceptibility is obtained from

$$
\chi=\sum_{x} \Gamma(x)=N_{\sigma}^{3}\left(\left\langle L^{2}\right\rangle-\langle\bar{L}\rangle^{2}\right)
$$

By the fluctuation-dissipation theorem it is related to the correlation function and hence it is also expected to diverge at $T_{\mathrm{c}}$.

\subsection{RESULTS ABOVE THE DECONFINEMENT TRANSITION}

As already mentioned we have performed a fit to the connected correlation function using eqs. (20) and (21) with $\bar{c}_{1}=0$ and the data we have measured on our $18^{3} \times 3,4$ and 5 lattices. The resulting $\xi / a$ values are shown in fig. 9. The error bars were obtained by the $\chi^{2}-95 \%$ confidence level method. The solid lines in fig. 9 are the best fits to the form

$$
(\xi / a)^{-1}=A\left(4 / g^{2}-4 / g_{c}^{2}\right)^{\nu}\left(1+B\left(4 / g^{2}-4 / g_{c}^{2}\right)^{0.5}\right),
$$

where the critical coupling $4 / g_{c}^{2}$ was taken from the corresponding $\langle\bar{L}\rangle$ fits and $\nu$ was fixed to its Ising value 0.63 . The data points are seen to be well in accord with

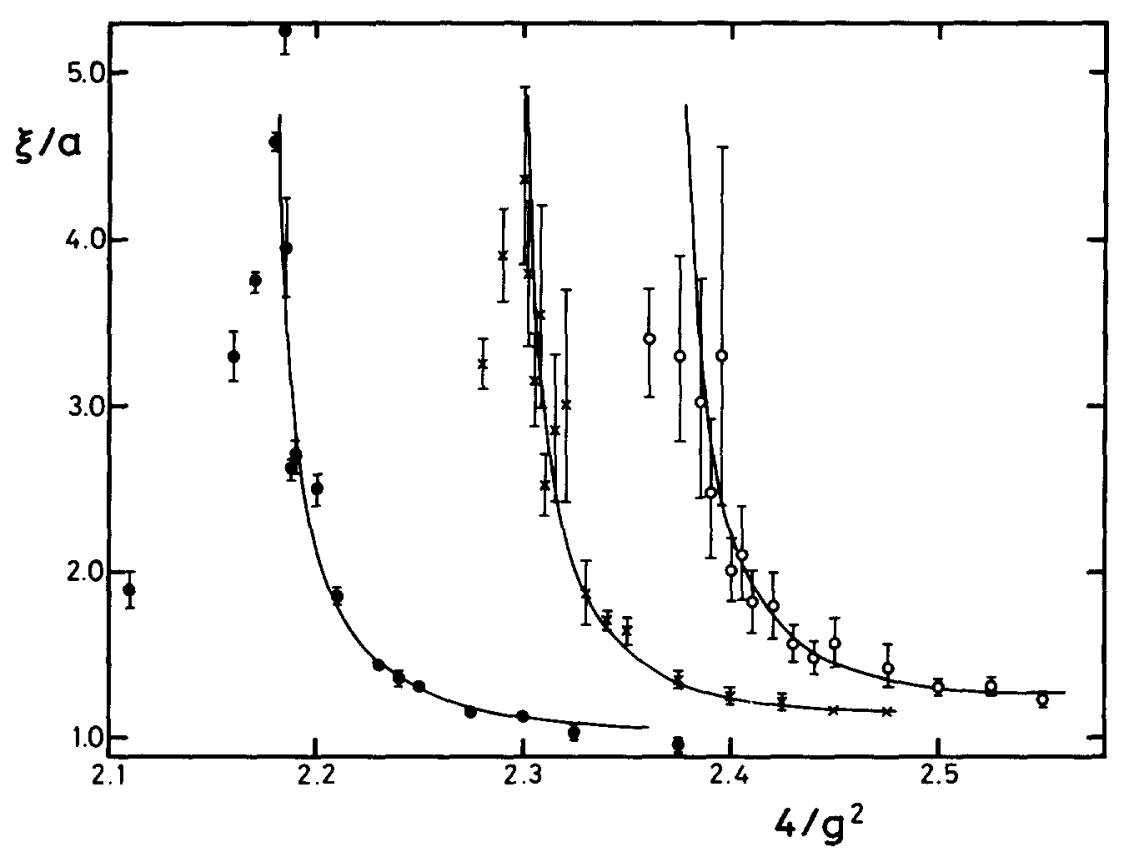

Fig. 9. Correlation length versus $4 / g^{2}$ on the $18^{3} \times 3(\bullet), 18^{3} \times 4(\times)$ and $18^{3} \times 5(0)$ lattices. The solid lines are best fits to eq. (23) with fixed $\nu=0.63$. 


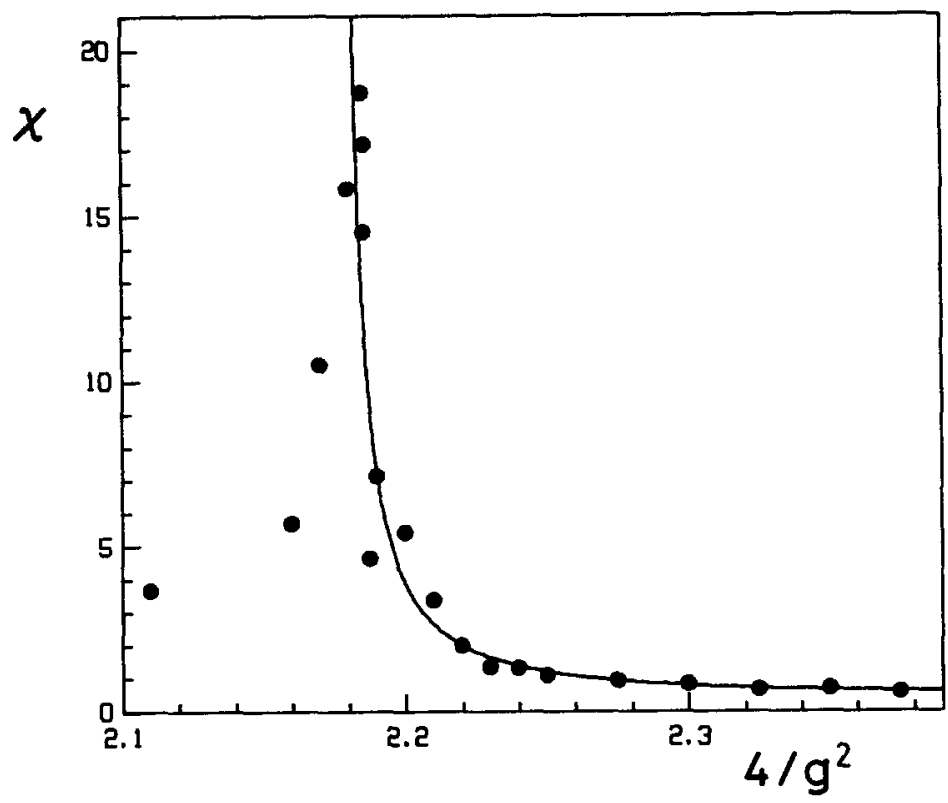

Fig. 10. Susceptibility versus $4 / \mathrm{g}^{2}$ on the $18^{3} \times 3$ lattice. The curve is a fit to eq. (24) with fixed $\gamma=1.24$.

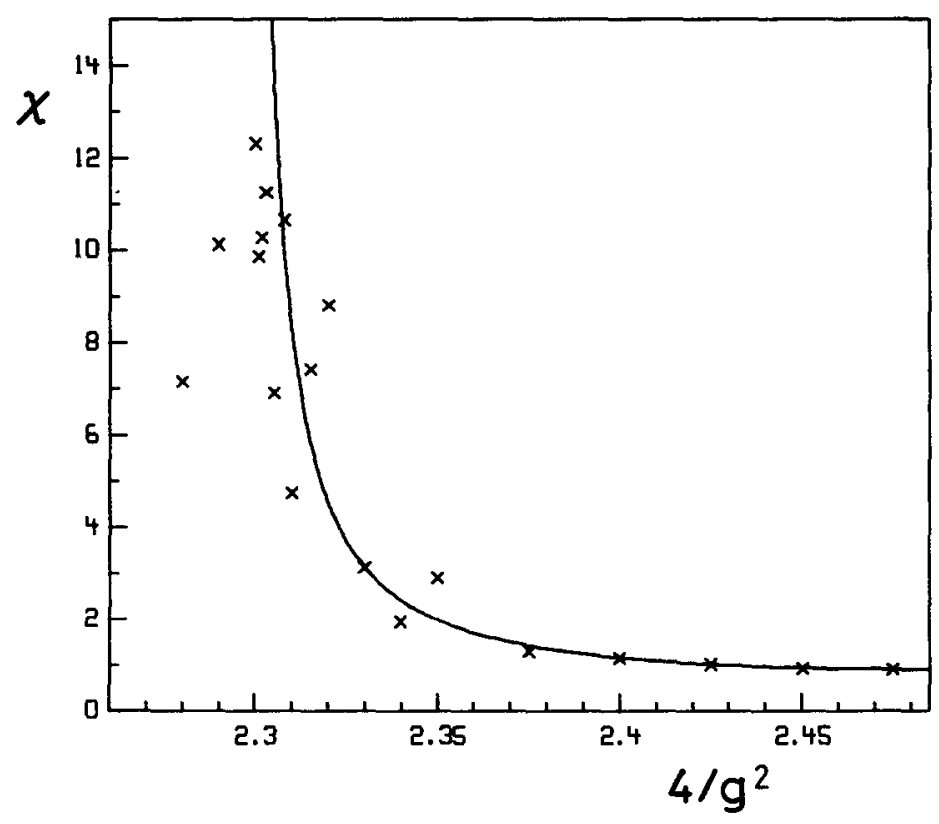

Fig. 11. As fig. 10, for the $18^{3} \times 4$ lattice. 


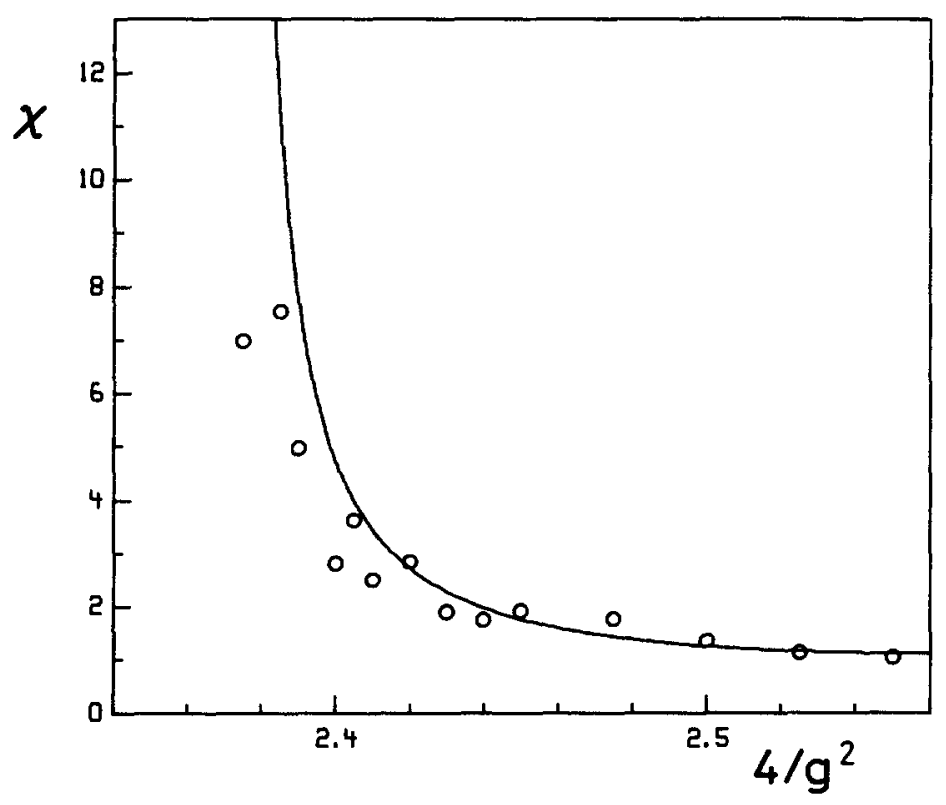

Fig. 12. As fig. 10 , for the $18^{3} \times 5$ lattice.

the fits using the universality conjecture. We have also tried fits to determine $\nu$ by directly fitting eq. (23) to our data; as a result of the large errors of $\xi$ near the transition point, this does not really lead to a conclusive determination.

In figs. 10-12 we present our data for the susceptibility measured on $18^{3} \times 3,4$ and 5 lattices, respectively. The errors are large and not included in the figures. As for the order parameter and the correlation length we have assumed a two-term fit form

$$
\chi^{-1}=A\left(4 / g^{2}-4 / g_{c}^{2}\right)^{\gamma}\left(1+B\left(4 / g^{2}-4 / g_{c}^{2}\right)^{0.5}\right),
$$

where we fix again the critical couplings to those obtained from the $\langle\bar{L}\rangle$ fits and $\gamma$ to the Ising model prediction $\gamma=1.24$. The resulting fits support the universality prediction, a direct determination of $\gamma$ is, due to the large errors of the data, not feasible.

\subsection{RESULTS FROM THE CONFINEMENT REGION}

Below the critical temperature we have performed additional measurements on $16^{3} \times 4,5$ and 6 lattices at $4 / g^{2}=2.24,2.25$ and 2.26, i.e. seen as a function of $T$ we have 9 data points. Of course, this transformation from the variable $4 / g^{2}$ to $T$ involves again the two-loop $\beta$-function, eq. (3) and may contain an error due to deviations from this asymptotic scaling law. We know already from our $T_{\mathrm{c}}$ de- 


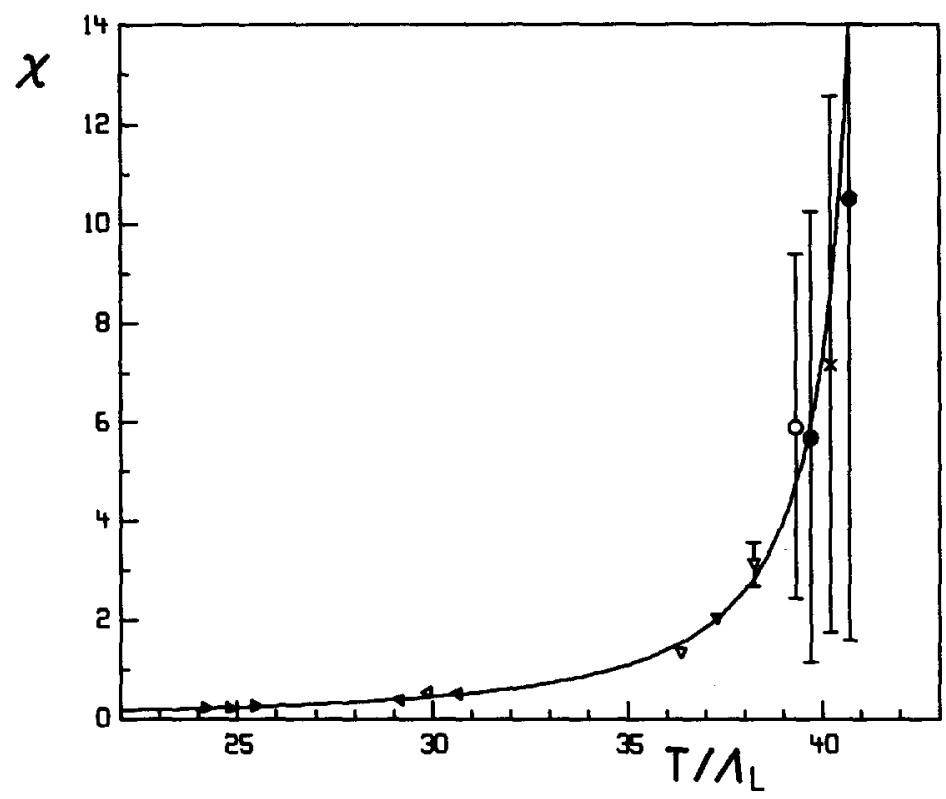

Fig. 13. Susceptibility versus temperature below $T_{\mathrm{c}}$ for $16^{3} \times 4(\nabla), 5(\triangleleft), 6(\triangleright)$ and $18^{3} \times 3(\bullet), 4(\times)$, $5(0)$ lattices.

terminations, that the error cannot be large. To minimize such effects further the data on the $16^{3} \times N_{\tau}$ lattices were taken at close $4 / g^{2}$ values. The inclusion of data from the $18^{3} \times N_{\tau}$ lattices in the broader interval $2.16 \leqslant 4 / \mathrm{g}^{2} \leqslant 2.36$ leads however to consistent results and confirms that these errors are not important. We considered four points from $18^{3} \times N_{\tau}$ lattices: $N_{\tau}=3,4 / g^{2}=2.16,2.17 ; N_{\tau}=4,4 / g^{2}=$ 2.28; $N_{\tau}=5,4 / g^{2}=2.36$.

We have plotted the data for the susceptibility $\chi$ in fig. 13 and fitted them according to

$$
\chi=A\left(T_{\mathrm{c}} / \Lambda_{\mathrm{L}}-T / \Lambda_{\mathrm{L}}\right)^{-\gamma}\left(1+B\left(T_{\mathrm{c}} / \Lambda_{\mathrm{L}}-T / \Lambda_{\mathrm{L}}\right)^{0.5}\right) .
$$

The same strong correlation between the value of the critical point $T_{\mathrm{c}} / \Lambda_{\mathrm{L}}$ and the critical exponent $\gamma$ is found like for the previous fits. Both the fits to the 9 points as those to the 13 including the $18^{3} \times N_{\tau}$ results lead to a flat $\chi^{2}$ function. We therefore fix $T_{\mathrm{c}}$ to be $T_{\mathrm{c}} / \Lambda_{\mathrm{L}}=41.8$ (the value on the $18^{3} \times 4$ lattice from the $\langle\bar{L}\rangle$ fit in sect. 2).

The fit shown in fig. 13 was obtained for fixed $\gamma=1.24$; evidently the data are compatible with the universality prediction.

The correlation function was then fitted according to eqs. (20) and (21). It turned out, that the coefficient $\overline{c_{1}}$ was negligible, so the fit was finally performed with 


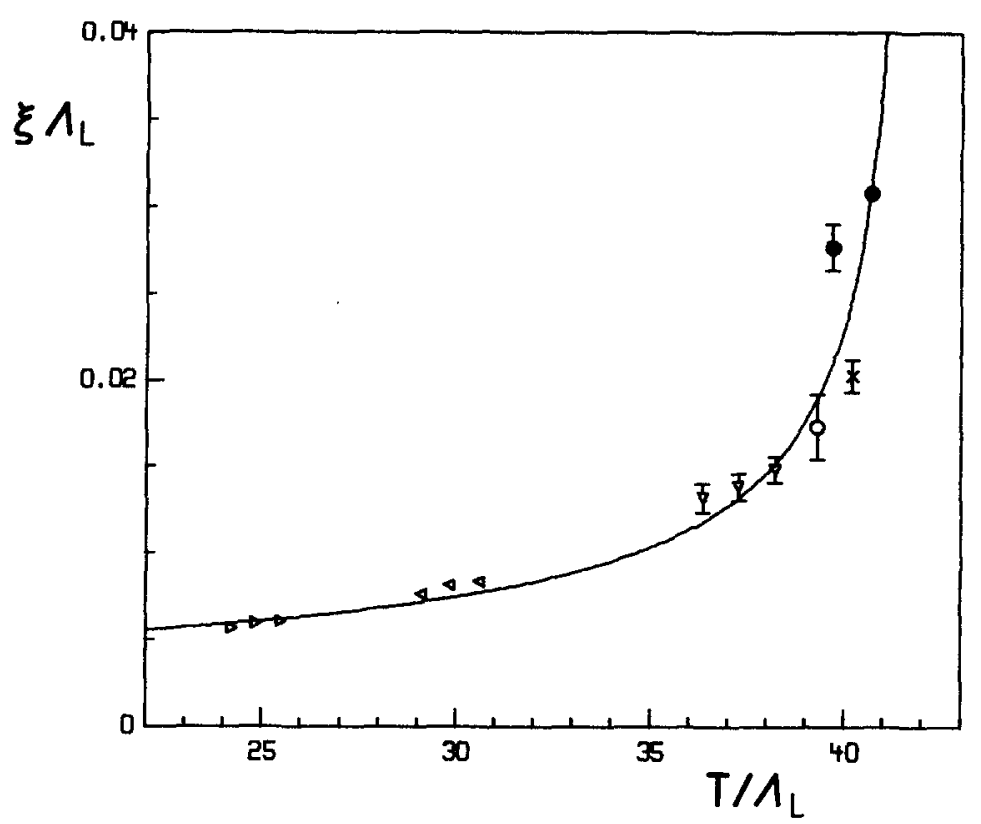

Fig. 14. Correlation length versus temperature below $T_{c}$, same notation as in fig. 13.

$\overline{c_{1}}=0$. The values for $\overline{c_{2}}$ were then transformed to obtain $\xi \Lambda_{\mathrm{L}}$ and $\sigma / \Lambda_{\mathrm{L}}^{2}$. In figs. 14 and 15 we show these results. One may now try two different ways to obtain the critical exponent $\nu$. Either we perform a fit for $\sigma / \Lambda_{\mathrm{L}}^{2}$

$$
\sigma / \Lambda_{\mathrm{L}}^{2}=A\left(T_{\mathrm{c}} / \Lambda_{\mathrm{L}}-T / \Lambda_{\mathrm{L}}\right)^{\nu}\left(1+B\left(T_{\mathrm{c}} / \Lambda_{\mathrm{L}}-T / \Lambda_{\mathrm{L}}\right)^{\theta}\right)
$$

or one for $\xi \Lambda_{\mathrm{L}}$

$$
\xi \Lambda_{\mathrm{L}}=\tilde{A}\left(T_{\mathrm{c}} / \Lambda_{\mathrm{L}}-T / \Lambda_{\mathrm{L}}\right)^{-\nu}\left(1+\tilde{B}\left(T_{\mathrm{c}} / \Lambda_{\mathrm{L}}-T / \Lambda_{\mathrm{L}}\right)^{\theta}\right),
$$

again with $\theta=0.5$. In the close vicinity to the critical temperature both expressions will lead to the same exponent $\nu$, however, because of relation (18) we have

$$
\sigma / \Lambda_{\mathrm{L}}^{2}=\left(T / \Lambda_{\mathrm{L}}\right) /\left(\xi \Lambda_{\mathrm{L}}\right),
$$

i.e. a different $T$ behaviour for the two quantities. Since we are dealing with a relatively large range for $T / \Lambda_{\mathrm{L}}\left(24 \leqslant T / \Lambda_{\mathrm{L}} \leqslant 40\right)$ the fit results will differ, also because the leading correction cannot be absorbed into a redefinition of $B \rightarrow \tilde{B}$ with $\theta=0.5$. On the other hand it is known that $\sigma$ remains finite at $T=0$ and so $\xi(T=0)=0$. We may therefore expect that the fit of $\xi \Lambda_{\mathrm{L}}$ away from the critical point becomes faster unreliable then that for $\sigma / \Lambda_{\mathrm{L}}^{2}$. One finds that the $\xi \Lambda_{\mathrm{L}}$ fit gives 


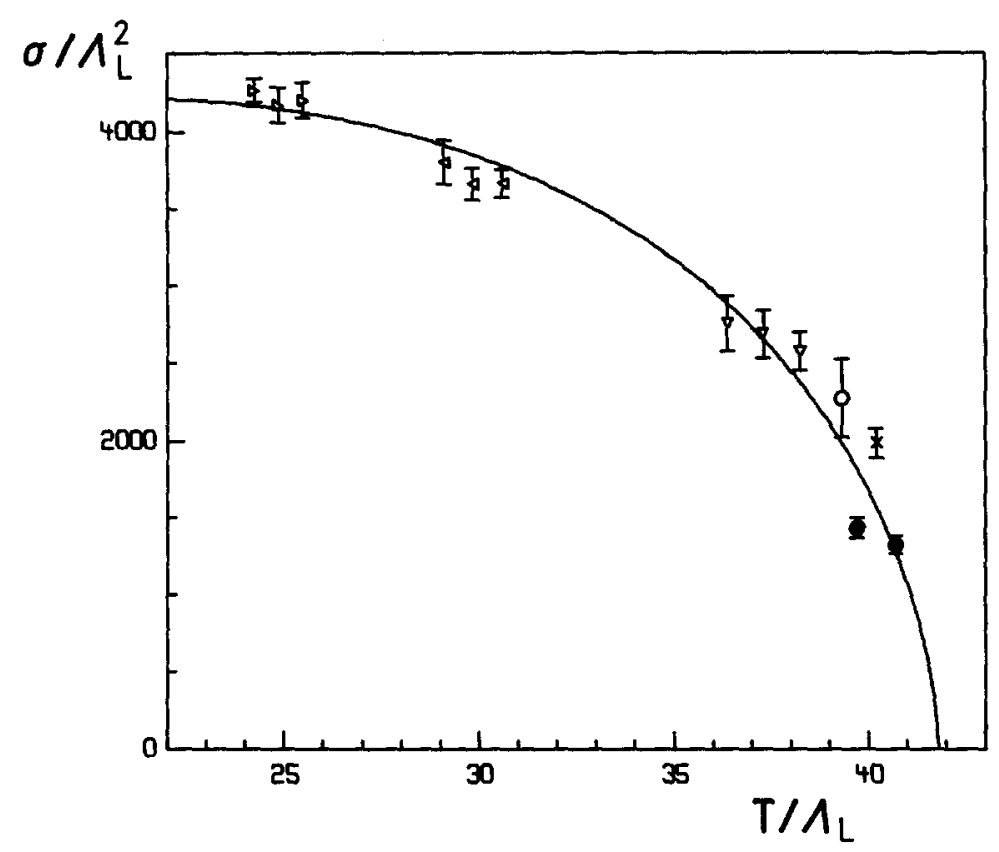

Fig. 15. String tension versus temperature, same notation as in fig. 13.

typically $30 \%$ smaller values for $\nu$ whereas the $\nu$-value from the fit to $\sigma / \Lambda_{\mathrm{L}}^{2}$ is close to the universality prediction $\nu=0.63$. In view of the large errors on the critical exponent $\nu$ from both fits, we show in figs. 14 and 15 only the curves for fixed $\nu=0.63$ and $T_{c}=41.8 \Lambda_{\mathrm{L}}$.

\section{Summary}

We have calculated the order parameter, the susceptibility, the correlation length and the energy density of the SU(2) Yang-Mills system on large lattices with high statistics. The resulting data are listed in tables 4-6 for the $18^{3} \times 3,4,5$ lattices and table 7 for the $16^{3} \times 4,5,6$ lattices. The points, which we calculated on an $8^{3} \times 3$ lattice for a demonstration of finite size effects are given in table 8.

Using these data, the critical exponent of the deconfinement order parameter was then determined. We find in all cases very good agreement with the universality prediction. In addition, we note that the fits have to include corrections-to-scaling terms and that there are strong correlations between the critical exponents and the critical couplings/or temperatures.

With the universality prediction for the critical exponent $\beta$ of the order parameter as input, the critical couplings $4 / g_{c}^{2}$ could be determined very accurately. From the 
TABLE 4

Numerical results from the $18^{3} \times 3$ lattice

\begin{tabular}{llcrrr}
\hline $4 / g^{2}$ & $T / \Lambda_{\mathrm{L}}$ & $\langle\bar{L}\rangle$ & $\chi$ & $\xi / a$ & $\varepsilon_{0} a^{4} \times 10^{3}$ \\
\hline 2.11 & 35.05 & $0.0196(17)$ & 3.05 & $1.90(10)$ & $1.79(32)$ \\
2.16 & 39.70 & $0.0459(48)$ & 5.70 & $3.30(16)$ & $3.51(34)$ \\
2.17 & 40.71 & $0.0800(66)$ & 10.50 & $3.76(07)$ & $5.21(37)$ \\
2.18 & 41.74 & $0.0956(81)$ & 15.84 & $4.59(05)$ & $6.11(31)$ \\
2.185 & 42.26 & $0.1151(92)$ & 18.72 & $5.26(15)$ & $8.09(58)$ \\
2.1855 & 42.31 & $0.1556(82)$ & 14.51 & $3.95(30)$ & $10.83(69)$ \\
2.186 & 42.37 & $0.1567(62)$ & 17.18 & & $10.87(46)$ \\
2.1875 & 42.53 & $0.1881(32)$ & 4.65 & $2.62(08)$ & $13.17(37)$ \\
2.19 & 42.79 & $0.1926(46)$ & 7.14 & $2.70(10)$ & $14.23(52)$ \\
2.20 & 43.87 & $0.2194(55)$ & 5.44 & $2.49(10)$ & $16.68(61)$ \\
2.21 & 44.99 & $0.2546(34)$ & 3.39 & $1.84(05)$ & $21.14(59)$ \\
2.22 & 46.12 & $0.2793(27)$ & 2.00 & & $24.77(52)$ \\
2.23 & 47.29 & $0.2970(14)$ & 1.36 & $1.43(01)$ & $26.39(44)$ \\
2.24 & 48.49 & $0.3080(20)$ & 1.34 & $1.36(05)$ & $27.87(58)$ \\
2.25 & 49.72 & $0.3207(15)$ & 1.11 & $1.31(01)$ & $29.60(40)$ \\
2.275 & 52.94 & $0.3501(11)$ & 0.96 & $1.16(02)$ & $33.60(35)$ \\
2.30 & 56.36 & $0.3691(12)$ & 0.84 & $1.13(02)$ & $34.85(34)$ \\
2.325 & 60.01 & $0.3905(07)$ & 0.67 & $1.02(04)$ & $37.45(33)$ \\
2.35 & 63.90 & $0.4047(12)$ & 0.70 & & $38.48(35)$ \\
2.375 & 68.04 & $0.4178(07)$ & 0.59 & $0.96(04)$ & $39.22(34)$ \\
$\infty$ & & 1.0 & & & 42.86 \\
\hline
\end{tabular}

asymptotic scaling relation, eq. (3) we find then

$$
\begin{array}{ll}
T_{\mathrm{c}} / \Lambda_{\mathrm{L}}=41.40 \pm_{0.30}^{0.25} & \text { for } N_{\tau}=3, \\
T_{\mathrm{c}} / \Lambda_{\mathrm{L}}=41.89 \pm_{0.18}^{0.15} & \text { for } N_{\tau}=4, \\
T_{\mathrm{c}} / \Lambda_{\mathrm{L}}=40.58 \pm_{0.53}^{0.38} & \text { for } N_{\tau}=5 .
\end{array}
$$

The errors are again determined with the $95 \%$ confidence level method. The results vary slightly less than those of Curzi and Trippiccione [15] and are consistent with the $\beta$-function measurements with the ratio method [23]. The values found for $T_{\mathrm{c}} / \Lambda_{\mathrm{L}}$ are also confirmed by the measured energy densities on the $N_{\tau}=3,4,5$ lattices. We moreover find that the dimensionless ratios $\varepsilon / \varepsilon_{\mathrm{SB}}$ show the same temperature dependence, apart from a small shift in $T$ of the $N_{\tau}=5$ ratio compared to the $N_{\tau}=3,4$ results, in accord with the shift in the $T_{c} / \Lambda_{\mathrm{L}}$ values seen in eqs. (29)-(31). To improve the $T_{\mathrm{c}}$ determination and test the scaling behaviour, one would have to perform a finite size scaling analysis; one could also try to reduce finite size effects by choosing other spatial boundary conditions [24]. 
TABLE 5

Numerical results from the $18^{3} \times 4$ lattice

\begin{tabular}{lccrrr}
\hline $4 / g^{2}$ & $T / \Lambda_{\mathrm{L}}$ & $\langle\bar{L}\rangle$ & $\chi$ & $\xi / a$ & $\varepsilon_{0} a^{4} \times 10^{3}$ \\
\hline 2.28 & 40.20 & $0.0552(54)$ & 7.16 & $3.26(16)$ & $1.01(20)$ \\
2.29 & 41.22 & $0.0679(75)$ & 10.13 & $3.90(29)$ & $1.44(21)$ \\
2.30 & 42.27 & $0.0986(79)$ & 12.32 & $4.36(57)$ & $2.16(21)$ \\
2.301 & 42.38 & $0.1109(60)$ & 9.88 & & $2.53(26)$ \\
2.302 & 42.48 & $0.1114(60)$ & 10.28 & $3.79(43)$ & $2.35(22)$ \\
2.303 & 42.59 & $0.1084(66)$ & 11.27 & & $2.39(27)$ \\
2.305 & 42.80 & $0.1324(44)$ & 6.92 & $3.15(28)$ & $3.32(22)$ \\
2.308 & 43.13 & $0.1365(55)$ & 10.66 & $3.54(65)$ & $3.39(26)$ \\
2.31 & 43.34 & $0.1517(30)$ & 4.75 & $2.52(19)$ & $3.71(17)$ \\
2.315 & 43.89 & $0.1566(36)$ & 7.42 & $2.87(46)$ & $3.86(15)$ \\
2.32 & 44.45 & $0.1701(60)$ & 8.83 & $3.01(71)$ & $4.84(25)$ \\
2.33 & 45.58 & $0.1988(33)$ & 3.12 & $1.87(21)$ & $5.78(26)$ \\
2.34 & 46.73 & $0.2120(17)$ & 1.95 & $1.70(07)$ & $6.32(21)$ \\
2.35 & 47.92 & $0.2239(30)$ & 2.90 & $1.64(10)$ & $7.26(27)$ \\
2.375 & 51.03 & $0.2537(16)$ & 1.30 & $1.35(05)$ & $8.36(27)$ \\
2.40 & 54.34 & $0.2743(13)$ & 1.15 & $1.25(05)$ & $8.79(23)$ \\
2.425 & 57.87 & $0.2893(12)$ & 1.02 & $1.21(05)$ & $9.10(23)$ \\
2.45 & 61.63 & $0.3022(09)$ & 0.93 & $1.16(02)$ & $9.77(17)$ \\
2.475 & 65.64 & $0.3157(08)$ & 0.91 & $1.16(02)$ & $9.90(23)$ \\
$\infty$ & & 1.0 & & & 11.52 \\
\hline
\end{tabular}

TABLE 6

Numerical results from the $18^{3} \times 5$ lattice

\begin{tabular}{lccrrr}
\hline $4 / g^{2}$ & $T / \Lambda_{\mathrm{L}}$ & \multicolumn{1}{c}{$\langle\bar{L}\rangle$} & \multicolumn{1}{c}{$\boldsymbol{\xi}$} & \multicolumn{1}{c}{$\xi / a$} & $\varepsilon_{0} a^{4} \times 10^{3}$ \\
\hline 2.36 & 39.31 & $0.0498(39)$ & 19.67 & $3.40(38)$ & $3.04(122)$ \\
2.375 & 40.83 & $0.0888(42)$ & 6.98 & $3.29(61)$ & $9.77(122)$ \\
2.385 & 41.86 & $0.1096(45)$ & 7.54 & $3.02(72)$ & $12.70(156)$ \\
2.39 & 42.39 & $0.1228(34)$ & 4.98 & $2.48(42)$ & $12.53(148)$ \\
2.395 & 42.93 & $0.1261(48)$ & 38.07 & $3.30(126)$ & $16.85(154)$ \\
2.40 & 43.47 & $0.1444(22)$ & 2.81 & $1.99(20)$ & $18.69(129)$ \\
2.405 & 44.03 & $0.1468(33)$ & 3.62 & $2.10(32)$ & $18.88(139)$ \\
2.41 & 44.58 & $0.1585(25)$ & 2.50 & $1.81(19)$ & $22.55(117)$ \\
2.42 & 45.72 & $0.1661(28)$ & 2.84 & $1.79(22)$ & $22.61(206)$ \\
2.43 & 46.88 & $0.1782(21)$ & 1.90 & $1.56(13)$ & $26.78(172)$ \\
2.44 & 48.08 & $0.1887(19)$ & 1.75 & $1.48(11)$ & $27.71(191)$ \\
2.45 & 49.31 & $0.1920(25)$ & 1.92 & $1.57(15)$ & $30.35(192)$ \\
2.475 & 52.51 & $0.2103(23)$ & 1.77 & $1.42(14)$ & $34.50(271)$ \\
2.50 & 55.93 & $0.2243(14)$ & 1.36 & $1.30(05)$ & $32.90(170)$ \\
2.525 & 59.58 & $0.2354(16)$ & 1.13 & $1.30(06)$ & $34.82(221)$ \\
2.55 & 63.46 & $0.2474(16)$ & 1.05 & $1.22(05)$ & $36.72(217)$ \\
$\infty$ & & 1.0 & & & 40.73 \\
\hline
\end{tabular}


TABLE 7

Numerical results from the $16^{3} \times N_{\tau}$ lattices

\begin{tabular}{cccccc}
\hline$N_{\tau}$ & $4 / g^{2}$ & $T / \Lambda_{\mathrm{L}}$ & $\chi$ & $\sigma a^{2}$ & $\xi / a$ \\
\hline \multirow{2}{*}{6} & 2.24 & 24.26 & $0.224(09)$ & $0.202(04)$ & $0.827(16)$ \\
& 2.25 & 24.86 & $0.226(13)$ & $0.187(05)$ & $0.889(26)$ \\
& 2.26 & 25.49 & $0.264(12)$ & $0.179(05)$ & $0.929(27)$ \\
5 & 2.24 & 29.10 & $0.398(25)$ & $0.180(07)$ & $1.113(43)$ \\
& 2.25 & 29.83 & $0.547(48)$ & $0.164(05)$ & $1.216(35)$ \\
& 2.26 & 30.59 & $0.515(37)$ & $0.157(05)$ & $1.277(37)$ \\
4 & 2.24 & 36.37 & $1.324(148)$ & $0.130(09)$ & $1.919(126)$ \\
& 2.25 & 37.29 & $2.015(187)$ & $0.121(07)$ & $2.068(120)$ \\
& 2.26 & 38.24 & $3.126(444)$ & $0.110(06)$ & $2.271(114)$ \\
\hline
\end{tabular}

TABLE 8

Numerical results from the $8^{3} \times 3$ lattice

\begin{tabular}{llllr}
\hline $4 / g^{2}$ & $T / \Lambda_{\mathrm{L}}$ & \multicolumn{1}{c}{$\langle\bar{L}\rangle$} & \multicolumn{1}{c}{$\chi$} & \multicolumn{1}{c}{$\varepsilon_{0} a^{4} \times 10^{3}$} \\
\hline 2.15 & 38.72 & $0.1177(96)$ & 2.73 & $5.44(113)$ \\
2.17 & 40.71 & $0.1652(150)$ & 3.25 & $10.60(197)$ \\
2.185 & 42.26 & $0.1941(84)$ & 3.40 & $14.89(147)$ \\
2.19 & 42.79 & $0.2133(106)$ & 3.85 & $17.81(143)$ \\
2.195 & 43.33 & $0.2348(81)$ & 2.83 & $18.84(131)$ \\
2.21 & 44.99 & $0.2578(101)$ & 3.05 & $21.46(189)$ \\
2.23 & 47.29 & $0.2950(78)$ & 2.19 & $26.25(173)$ \\
2.25 & 49.72 & $0.3307(41)$ & 1.22 & $31.60(145)$ \\
$\infty$ & & 1.0 & & 42.25 \\
\hline
\end{tabular}

The research of J.E., K.K. and T.N. has been partly supported by the Deutsche Forschungsgemeinschaft; K.K. was during the major part of this work an Alexander von Humboldt fellow. J.E. thanks SCRI for its support and its members for the warm hospitality extended to him during his stay at SCRI. He acknowledges helpful discussions with $\mathrm{D}$. Duke and G. Bhanot about the three-dimensional Ising model. We are grateful to the computer center of the Bochum University for the large amount of Cyber 205 time, which was necessary to obtain our data.

\section{Appendix}

The errors of our raw data were estimated by the coarse-graining method [25, 26]. The average value of an observable $x$ from any $m$ consecutive Monte Carlo measurements after thermalization is defined as

$$
\bar{x}(m)=\frac{1}{m} \sum_{i=1}^{m} x_{i} .
$$


If $V(\bar{x}(m))$ is the variance of $\bar{x}(m)$, then

$$
\boldsymbol{\sigma}^{2}(m)=m V(\bar{x}(m))
$$

would be the variance of a single measurement $x_{i}$ and even be independent of $m$, provided all $x_{i}$ were independent. For sufficiently large $m$, say $m \geq N_{\mathrm{a}}$, we may expect that

$$
\sigma^{2}(m) \simeq \sigma^{2}(\infty)
$$

up to corrections of the order $1 / m$. Here $N_{\mathrm{a}}$ is the autocorrelation "time".

Instead of directly measuring the correlations among the $x_{i}$ and determining $N_{\mathrm{a}}$ by that, we have estimated $\sigma^{2}(\infty)$ from $V(\bar{x}(m))$ estimates for sufficiently large $m$. From the total number of, say, 40000 measurements we built $10 \bar{x}(4000)_{i}, 20$ $\bar{x}(2000)_{i}$ and $40 \bar{x}(1000)_{i}$ etc. This procedure was repeated for all observables. We found, that $V(\bar{x}(1000)) / V(\bar{x}(2000))$ is about $2 \pm 0.5$ off and $2 \pm 1$ near the critical point. The final $\sigma^{2}(\infty)$ was estimated from $\sigma^{2}(1000)$ and $\sigma^{2}(2000)$. The error of the average value of all measurements is then given by

$$
\Delta \bar{x}(n)=\frac{\sigma(\infty)}{\sqrt{n}},
$$

where $n$ is the total number of measurements. A crude estimate of the autocorrelation "time" $N_{\mathrm{a}}$ is obtained, if we approximate

$$
V(\bar{x}(n)) \simeq V(\bar{x}(1)) /\left(n / N_{\mathrm{a}}\right),
$$

or

$$
N_{\mathrm{a}} \simeq \sigma^{2}(\infty) / \sigma^{2}(1),
$$

since $n / N_{\mathrm{a}}$ is the effective number of independent measurements. We find, that $N_{\mathrm{a}}$ is about 100 , growing up to about 1000 in the close vicinity of the critical point.

\section{References}

[1] A.M. Polyakov, Phys. Lett. 72B (1978) 477

[2] L. Susskind, Phys. Rev. D20 (1979) 2610

[3] L. McLerran and B. Svetitsky, Phys. Lett. 98B (1981) 195

[4] J. Kuti, J. Polónyi and K. Szlachányi, Phys. Lett. 98B (1981) 199

[5] L. McLerran and B. Svetitsky, Phys. Rev. D24 (1981) 450

[6] J. Polónyi and K. Szlachányi, Phys. Lett. 110B (1982) 395

[7] B. Svetitsky and L.G. Yaffe, Nucl. Phys. B210 [FS6] (1982) 423

[8] M. Gross, Phys, Lett. 132B (1983) 125

[9] J.C. Le Guillou and J. Zinn-Justin, Phys. Rev. Lett. 39 (1977) 95; Phys. Rev. B21 (1980) 3976

[10] J.C. Le Guillou and J. Zinn-Justin, J. Physique Lett. 46 (1985) L137

[11] J.-H. Chen, B.G. Nickel and M.E. Fischer, Phys. Rev. Lett. 48 (1982) 630; M.E. Fisher and J.-H. Chen, Oxford preprint (1985) 
[12] G. Bhanot, D. Duke and R. Salvador, Phys. Rev. B33 (1986) 7841

[13] J. Kogut, M. Stone, H.W. Wyld, W.R. Gibbs, J. Shigemitsu, S.H. Shenker and D.K. Sinclair, Phys. Rev. Lett. 50 (1983) 393

[14] R.V. Gavai and H. Satz, Phys. Lett. 145B (1984) 248

[15] G. Curzi and R. Tripiccione, Phys. Lett. 151B (1985) 145

[16] J. Kiskis, Phys. Rev. D33 (1986) 2380

[17] B.G. Nickel, in Phase transitions, Cargese 1980, eds. M. Levy, J.C. Le Guillou and J. Zinn-Justin (Plenum, New York, 1982)

[18] J. Engels, F. Karsch, I. Montvay and H. Satz, Nucl, Phys. B205 [FS5] (1982) 545

[19] M. Nauenberg, T. Schalk and R. Brower, Phys. Rev. D24 (1981) 548;

B. Lautrup and M. Nauenberg, Phys. Rev. Lett. 45 (1980) 1755

[20] J. Engels, F. Karsch and H. Satz, Nucl. Phys. B205 [FS5] (1982) 239

[21] K. Kanaya and H. Satz, Phys. Rev. D34 (1986) 3193

[22] M. Lüscher, K. Symanzik and P. Weisz, Nucl. Phys. B173 (1980) 365;

M. Lüscher, Nucl. Phys. B180 (1980) 317

[23] U. Heller and F. Karsch, Phys. Rev. Lett. 54 (1985) 1765

[24] A. Gonzalez-Arroyo and M. Okawa, Phys. Rev. D27 (1983) 2397

[25] W.W. Wood, Physics of simple liquids (Wiley, New York 1968) ch. 5;

R. Friedberg and J.E. Cameron, J. Chem. Phys. 52 (1970) 6049;

D.P. Landau, Phys. Rev. B13 (1976) 2997

[26] J. Ambjørn, A. Hey and S. Otto, Nucl. Phys. B210 (1982) 347;

G. Daniell, A. Hey and J. Mandula, Phys. Rev. D30 (1984) 2230 